\title{
Investigation of the Sound Generation Mechanisms for In-duct Orifice Plates
}

\author{
Fuyang Tao \\ The Hong Kong University of Science and Technology, \\ Clear Water Bay, Kowloon, Hong Kong, China. \\ Phillip Joseph \\ University of Southampton, Southampton, Hampshire SO17 1BJ, UK. \\ Xin Zhang* \\ The Hong Kong University of Science and Technology, \\ Clear Water Bay, Kowloon, Hong Kong SAR, China. \\ Oksana Stalnov \\ Israel Institute of Technology, Haifa 32000, Israel. \\ Matthias Siercke and Henning Scheel \\ Interior \& Near Field Noise, AIRBUS Operations GmbH, Hamburg, 21129, Germany.
}

(Dated: June 10, 2017) 


\begin{abstract}
Sound generation due to an orifice plate in a hard-walled flow duct which is commonly used in air distribution systems (ADS) and flow meters is investigated in this work. The aim is to provide an understanding of this noise generation mechanism based on measurements of the source pressure distribution over the orifice plate. A simple model is described that relates the broadband in-duct sound field to the surface pressure cross spectrum on both sides of the orifice plate. The model is based on the assumption that, following Curles acoustic analogy, the noise radiated from the orifice plate can be represented in terms of axial dipole sources distributed over the surface of the orifice plate with the appropriate correlation characteristics.

This work describes careful measurements of the surface pressure cross spectrum over the orifice plate from which the surface pressure distribution and correlation length is deduced. This information is then used in the model to predict the radiated in-duct sound field. Agreement within $3 \mathrm{~dB}$ between the predicted and directly measured sound fields is obtained, providing direct confirmation that the surface pressure fluctuations acting over the orifice plates are the main noise sources. Based on the model developed in this work, the contributions to the sound field from different radial locations of the orifice plate are calculated. It is shown that the sound source distributions on the upstream and downstream surface of the orifice plate are observed to be similar in both their radial variation and level in the low frequency range where only plane waves propagate. The surface pressure is shown to follow a $U^{3.9}$ velocity scaling law and the area over which the surface sources are correlated follows a $U^{1.8}$ velocity scaling law, as predicted in previous work by other researchers.
\end{abstract}

PACS numbers: PACS: 43.28.Ra

\footnotetext{
*aexzhang@ust.hk; Corresponding author.
} 


\section{INTRODUCTION}

Orifice plates as shown in Fig. 2 are widely used in air distribution systems (ADS) of aircraft, automotive systems and buildings, to control the flow rate. However, pressure loss is achieved by causing the flow around it to become unsteady, which results in unwanted noise generation. The unsteady flow interacting with the orifice plate can become the dominant noise source in the ADS. It is therefore important to understand the noise generation mechanisms of orifice plate to enable its prediction and to assist the design of quieter duct systems.

Previous researchers have measured the in-duct acoustic and flow field to investigate the noise generation mechanisms of in-duct orifice plates. Agarwal $[7,8]$, for example, has performed a series of measurements to investigate the sound generation mechanisms of an orifice plate based on the pressure spectra measured on the duct wall. These wall pressure measurements revealed five different flow regions, as shown in Fig. 1. The highest turbulence level was found to occur in the separation region just upstream and downstream of the orifice plate. Agarwal concluded that the dominant noise sources occur in this region of highest turbulence. To estimate the length of the separation region, Agarwal and Bull $[4,6]$ measured the wall shear stress using a stress fence gauge and concluded that the length of the separation region is about ten times the height of the orifice plate (i.e., difference between outer and inner radii). Similar conclusions about the length of the separation region were also made by Durst and Wang[1], Nail [2] and Feng et al. [3]. In the separation region, Agarwal's experiment shows that the wall pressure is dominated by hydrodynamic pressure fluctuations whose spectra could be collapsed on the dynamic pressure evaluated at the centre of the orifice plate. In the upstream and downstream regions beyond the separation region, acoustic pressure fluctuations were found to be dominant.

Kerschen and Johnston [10-12] investigated experimentally the properties of orifice plate noise generation based on modal analysis of the sound field in the duct. They concluded that orifice plate noise is generated by turbulence in the jet flow passing through the centre of the orifice plate. They stated without verification that higher order acoustic modes were due to excitation by coherent flow structures near the jet flow. They also found that the modal pressure spectra were determined by the ratio between the Helmholtz number $k a$ (where $a$ is the duct radius, $k$ is the wave number) and the Strouhal number $f L_{c} / U$ (where $L_{c}$ is the 


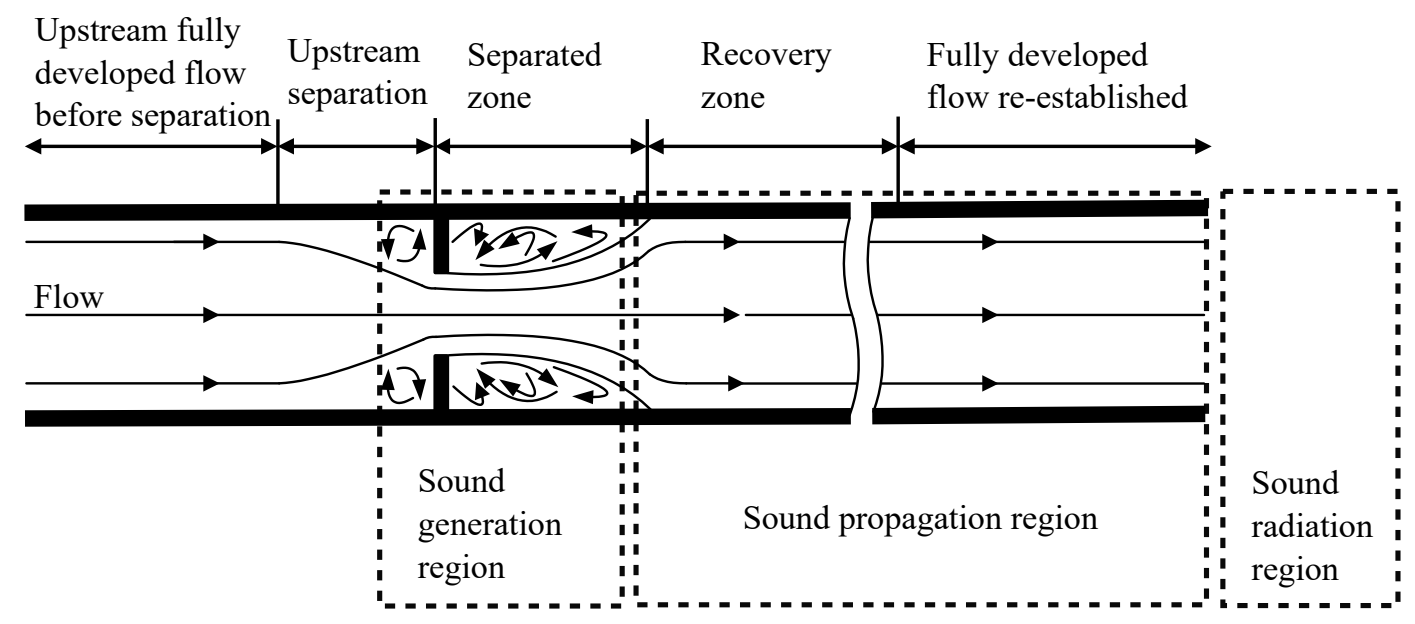

FIG. 1. Different flow regions in a duct with an orifice plate [8].

characteristic dimension of the orifice plate, $f$ is frequency and $U$ is mean flow speed), which govern acoustic modal propagation and the velocity spectral shape, respectively. When this ratio is small, the modal pressure spectra fall off rapidly with increasing frequency. When this ratio is large, the amplitude of each mode is approximately equal.

A semi-empirical model to predict the sound power due to an orifice plate installed near the end of a duct was developed by Gordon [14, 15] based on the assumption that the sources were located over the surface of the orifice plate. The main innovation in his model is the assumption that the surface pressure fluctuations, and hence total rms fluctuating force, acting on the orifice plate are proportional to the steady force acting across it. This steady force was determined from the pressure drop across the orifice plate and the cross-sectional area of the duct. Based on these same assumptions, Nelson and Morfey [13], Oldham and Ukpoho [16], Kårekull et al. [17] also developed similar models to predict the sound power generated by in-duct orifice plate.

This review of the previous work shows that there is still not complete agreement about the precise mechanism and distribution of the aerodynamic sources responsible for orifice plate noise. In this work, a model is developed to describe the relationships between the pressure fluctuations over the surface of the orifice plate and the radiated in-duct sound field. The model is based on the assumption that the fluctuating pressure difference across the orifice plate can be modeled as an axial dipole distribution over its surface. The model is then used in combination with careful measurements of the surface pressure two-point cross spectral density (CPSD) to predict the radiated in-duct noise and also to determine 


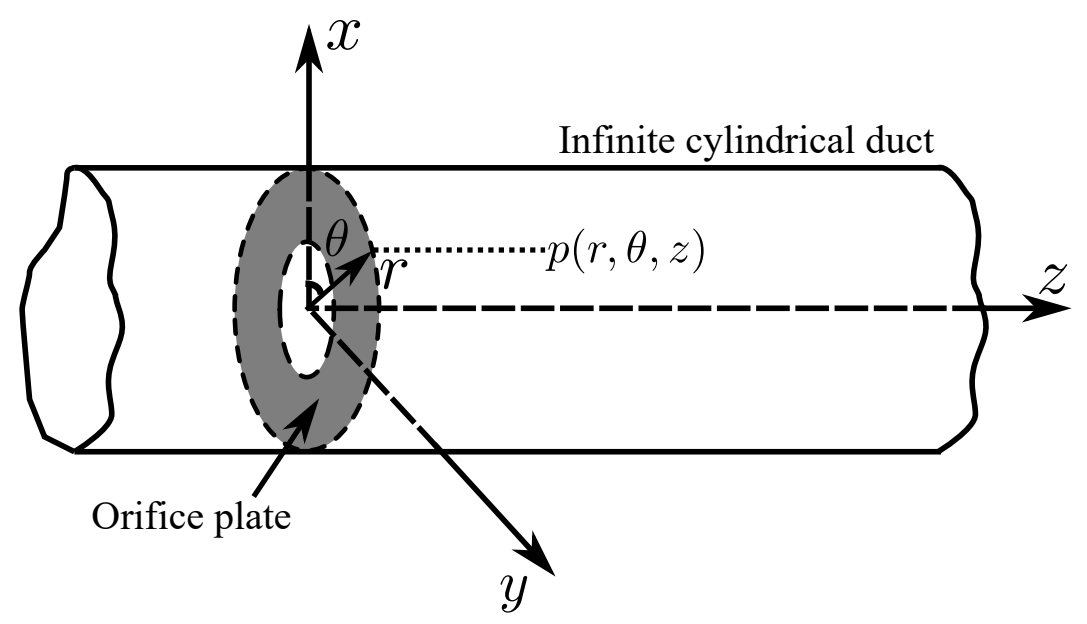

FIG. 2. Illustration of an orifice plate in an infinite cylinder and cylindrical coordinates system used for theoretical developments.

the distribution of the source strength over the surface of the orifice plate. Predictions of the radiated sound field are then compared to direct measurements using far field microphones.

\section{SURFACE PRESSURE MODEL FOR ORIFICE PLATE NOISE}

\section{A. Sound field in a cylindrical duct}

The basic principles of noise propagation in a hard walled cylindrical duct is discussed in this section. A cylindrical coordinate system $(r, \theta, z)$ is shown in Fig. 2. The acoustic pressure $p(\mathbf{x}, t)$ at a point $\mathbf{x}(r, \theta, z)$ in the duct, neglecting the effects of flow (since the Mach number $M<0.1$ for a typical ADS, satisfies the homogeneous wave equation of the form

$$
\nabla^{2} p(\mathbf{x}, t)-\frac{1}{c_{0}^{2}} \frac{\partial^{2} p(\mathbf{x}, t)}{\partial t^{2}}=0,
$$

where $c_{0}$ is the speed of sound. The sound field can be expressed as the sum of modal components $p=\sum_{m=-\infty}^{\infty} \sum_{n=0}^{\infty} p_{m n}$, where $(m, n)$ are the circumferential and radial mode indices, respectively. At a single frequency a single mode is of the form,

$$
p_{m n}(r, \theta, z, t)=A_{m n} \Psi_{m n}(r, \theta) e^{-i\left(k_{z m n} z-\omega t\right)},
$$

where $A_{m n}$ are the pressure amplitudes of mode $(m, n), k_{z m n}$ are the modal axial wave numbers, and $\Psi_{m n}(r, \theta)$ are the normalized mode shape functions of the form, 


$$
\Psi_{m n}(r, \theta)=\frac{J_{m}\left(k_{r m n} r\right)}{N_{m n}} e^{i m \theta}
$$

which satisfies $\left(\nabla_{\perp}^{2}+k_{r m n}^{2}\right) \Psi_{m n}(r, \theta)=0$ and the hard-walled duct boundary condition, and $k_{r m n}$ is the duct eigenvalue for mode $(m, n)$. In a hard walled duct $k_{r m n}$ is given by $j_{m n}^{\prime} / a$, where $j_{m n}^{\prime}$ is the $n^{\text {th }}$ stationary value of the Bessel function $J_{m}$ of order $m$ of the first kind and $N_{m n}$ is the normalisation constant, defined to ensure the normalisation condition over the duct cross-sectional area $S(r, \theta)$,

$$
\int_{S}\left|\Psi_{m n}(r, \theta)\right|^{2} d S(r, \theta)=1
$$

and is given by

$$
N_{m n}^{2}=\left\{\begin{array}{r}
A J_{m}^{2}\left(k_{r m n} a\right) \text { for } m=0, n=0 \\
A\left(1-\frac{m^{2}}{\left(k_{r m n} a\right)^{2}}\right) J_{m}^{2}\left(k_{r m n} a\right) \text { for } m \neq 0, n \neq 0
\end{array}\right.
$$

where $A$ is the duct cross sectional area $\pi a^{2}$.

\section{B. Development of surface pressure model for orifice plate noise}

According to Curle's theory for aerodynamic sound generation from solid surfaces [18], noise sources due to a turbulent flow distributed over a solid surface can be represented by a distribution of dipole sources orientated normal to the surface. As the orifice plate is perpendicular to the axial direction of the duct, the acoustic sources due to the orifice plate can be represented by acoustic dipole sources aligned in the axial direction distributed over the surfaces of the orifice plate. The dipole source strength is determined from the unsteady aerodynamic loading on the orifice plate, resulting from interaction between the unsteady flow and both sides of the orifice plate.

In this section, an expression is derived for the sound field induced by an axial dipole source distribution in an infinite cylindrical hard-walled duct in which the effects of flow can be ignored (since $M<0.1$ ). The time-varying pressure $p(\mathbf{x}, t)$ at any point $\mathbf{x}(r, \theta, z)$ in the cylindrical duct can be calculated from the Green's function solution to the wave equation 1 , 


$$
p(\mathbf{x}, t)=\int_{-\infty}^{+\infty} \int_{S_{r}} \mathbf{f}\left(\mathbf{x}_{s}, \tau\right) \cdot \nabla G\left(\mathbf{x}, t \mid \mathbf{x}_{s}, \tau\right) d S_{r}\left(\mathbf{x}_{s}\right) d \tau
$$

where $\mathbf{f}\left(\mathbf{x}_{s}, \tau\right)$ is the unsteady aerodynamic loading (i.e., net force) per unit area at the source point $\mathbf{x}_{s}\left(r_{s}, \theta_{s}, z_{s}\right)$ on the orifice plate surface $S_{r}$ at time $\tau$ and may be determined from the difference in unsteady pressures acting on both sides of the orifice plate, $\mathbf{f}\left(\mathbf{x}_{s}, \tau\right)=$ $\left(p_{s}^{-}\left(\mathbf{x}_{s}, \tau\right)-p_{s}^{+}\left(\mathbf{x}_{s}, \tau\right)\right) \hat{\mathbf{z}}$, where $p_{s}^{ \pm}\left(\mathbf{x}_{s}, \tau\right)$ refer to the surface pressure distributions over the upstream and downstream facing sides and $\hat{\mathbf{z}}$ is the unit vector in the duct axis direction $z$. A Green's function solution for an infinite, hard-walled cylindrical duct without flow can be expressed as [19]

$$
G\left(\mathbf{x}, t \mid \mathbf{x}_{s}, \tau\right)=\frac{i}{4 \pi} \sum_{m, n} \frac{\psi_{m n}\left(r_{s}\right) \psi_{m n}^{*}(r)}{N_{m n}^{2}} \int_{-\infty}^{+\infty} \frac{e^{i \omega(t-\tau)} e^{-i k_{z m n}\left(z-z_{s}\right)}}{k_{z m n}} d \omega
$$

As the orifice plate is installed perpendicular to the duct axis, $\mathbf{f}\left(\mathbf{x}_{s}, \tau\right) \cdot \nabla G\left(\mathbf{x}, t \mid \mathbf{x}_{s}, \tau\right)=$ $f\left(\mathbf{x}_{s}, \tau\right) \frac{\partial G}{\partial z_{s}}$, where $f\left(\mathbf{x}_{s}, \tau\right)$ is the magnitude of $\mathbf{f}\left(\mathbf{x}_{s}, \tau\right)$. The sound field induced by the orifice plate may therefore be expressed by

$$
p(\mathbf{x}, t)=\frac{1}{2 \pi} \int_{-\infty}^{\infty} \int_{S_{r}} f\left(\mathbf{x}_{s}, \tau\right) \sum_{m=-\infty}^{\infty} \int_{-\infty}^{\infty} g_{m}\left(\mathbf{x}_{s}, z, r, \omega\right) e^{i m \theta} e^{i \omega(t-\tau)} d \omega d S_{r}\left(\mathbf{x}_{s}\right) d \tau
$$

where $g_{m}$ is the component of the Green function associated with the spinning mode $m$ of the form,

$$
g_{m}\left(\mathbf{x}_{s}, z, r, \omega\right)=\frac{1}{2} \sum_{n=0}^{\infty} \frac{\psi_{m n}(r) \psi_{m n}^{*}\left(r_{s}\right) e^{-i m \theta_{s}}}{N_{m n}^{2}} e^{-i k_{z m n}\left(z-z_{s}\right)}
$$

Eq. (8) can be written in the frequency domain as

$$
p(\mathbf{x}, \omega)=\int_{S_{r}} f\left(\mathbf{x}_{s}, \omega\right) \sum_{m=-\infty}^{\infty} g_{m}\left(\mathbf{x}_{s}, z, r, \omega\right) e^{i m \theta} d S_{r}\left(\mathbf{x}_{s}\right)
$$

Since the noise from the orifice plate is broadband and therefore has a continuous spectrum, it can be expressed as the Power Spectral Density (PSD) of the acoustic pressure, defined by 


$$
S_{p p}(\mathbf{x}, \omega)=\lim _{T \rightarrow \infty} \frac{\pi}{T} E\left\{|p(\mathbf{x}, \omega)|^{2}\right\}
$$

where $E\{\}$ denotes expectation and $T$ denotes the time duration over which the Fourier transforms of the acoustic pressure $p(\omega)$ are taken. Substituting Eq. 10 into Eq. 11 gives

$$
\begin{aligned}
S_{p p}(\mathbf{x}, \omega)=\int_{S_{r}} \int_{S_{r}^{\prime}} & \sum_{m=-m_{0}}^{m_{0}} \sum_{m^{\prime}=-m_{0}}^{m_{0}} S_{f f}\left(\mathbf{x}_{s}, \mathbf{x}_{s}^{\prime}, \omega\right) \\
& \times g_{m}\left(\mathbf{x}_{s}, z, r, \omega\right) g_{m^{\prime}}^{*}\left(\mathbf{x}_{s}^{\prime}, z, r, \omega\right) e^{i m \theta-i m^{\prime} \theta} d S_{r}\left(\mathbf{x}_{s}\right) d S_{r}\left(\mathbf{x}_{s}{ }^{\prime}\right),
\end{aligned}
$$

where

$$
S_{f f}\left(\mathbf{x}_{s}, \mathbf{x}_{s}^{\prime}, \omega\right)=\lim _{T \rightarrow \infty} \frac{\pi}{T} E\left\{f\left(\mathbf{x}_{s}, \omega\right) f^{*}\left(\mathbf{x}_{s}^{\prime}, \omega\right)\right\}
$$

is the cross spectral density of the fluctuating force acting on the orifice plate.

At frequencies below the first modal cut-on frequency, only the plane wave can propagate in the duct. In this frequency range, Eq. 12 simplifies to

$$
S_{p p}(\mathbf{x}, \omega)=\frac{1}{4 A^{2}} \int_{S_{r}} \int_{S_{r}^{\prime}} S_{f f}\left(\mathbf{x}_{s}, \mathbf{x}_{s}^{\prime}, \omega\right) d S_{r}\left(\mathbf{x}_{s}\right) d S_{r}\left(\mathbf{x}_{s}^{\prime}\right)
$$

which represents the surface area average of the cross spectrum of the unsteady force distribution. Note the absence of receiver position $\mathbf{x}$ on the right hand side of this expression since the acoustic field is uniform in the infinite duct at all frequencies below the first cut-on frequency and hence independent of $\mathbf{x}$. At frequencies above the first modal cut-on frequency, higher order modes are excited, leading to,

$$
\begin{aligned}
S_{p p}(\mathbf{x}, \omega)=\frac{1}{4} \int_{S_{r}} \int_{S_{r}^{\prime}} & \sum_{m=-m_{0}}^{m_{0}} \sum_{n=0}^{n_{0}} \sum_{m^{\prime}=-m_{0}}^{m_{0}} \sum_{n^{\prime}=0}^{n_{0}} S_{f f}\left(\mathbf{x}_{s}, \mathbf{x}_{s}^{\prime}, \omega\right) \frac{\psi_{m n}(r) \psi_{m n}^{*}\left(r_{s}\right)}{N_{m n}^{2}} \\
& \times \frac{\psi_{m^{\prime} n^{\prime}}^{*}(r) \psi_{m^{\prime} n^{\prime}}\left(r_{s}^{\prime}\right)}{N_{m^{\prime} n^{\prime}}^{2}} e^{i m\left(\theta-\theta_{s}\right)} e^{-i m^{\prime}\left(\theta-\theta_{s}^{\prime}\right)} d S_{r}\left(\mathbf{x}_{s}\right) d S_{r}\left(\mathbf{x}_{s}^{\prime}\right) .
\end{aligned}
$$

Assuming that the unsteady loading on the two sides of the orifice plate due to flow interaction are uncorrelated, Eqs. 14 and 15 for the unsteady force cross spectra can be expressed 
as the sum of the pressure cross spectra on both sides,

$$
S_{f f}\left(\mathbf{x}_{s}, \mathbf{x}_{s}^{\prime}, \omega\right)=S_{p p}^{+}\left(\mathbf{x}_{s}, \mathbf{x}_{s}{ }^{\prime}, \omega\right)+S_{p p}^{-}\left(\mathbf{x}_{s}, \mathbf{x}_{s}{ }^{\prime}, \omega\right),
$$

where the superscripts \{\}$^{+}$and \{\}$^{-}$represent the surface pressure CPSD on the upstream and downstream surfaces of the orifice plate respectively, given by

$$
S_{p p}^{ \pm}\left(\mathbf{x}_{s}, \mathbf{x}_{s}^{\prime}, \omega\right)=\lim _{T \rightarrow \infty} \frac{\pi}{T} E\left\{p_{s}^{ \pm *}\left(\mathbf{x}_{s}, \omega\right) p_{s}^{ \pm}\left(\mathbf{x}_{s}^{\prime}, \omega\right)\right\}
$$

Unfortunately it was not possible to make pressure measurements on both sides of the orifice plate simultaneously due to the way in which channels were printed into the orifice plate. However, we believe that the assumption of uncorrelated pressures between the two sides of the orifice plate is justified because, as shown in Fig. 8, the coherence drops almost to zero for separation distances greater than $10 \mathrm{~mm}$ for measurements made on the same side. Furthermore, no physical mechanism exists by which flow structures can excite both sides of orifice plate coherently. Even coherence values of up 0.1 between the two sides would only lead to very small increases in the pressure noise radiation (about 1dB).

Using Eqs. 14 and 15, the PSD of the acoustic field in the cylindrical hard-walled duct can be obtained once the fluctuation pressure cross spectra over both sides of the orifice plate are known.

\section{EXPERIMENTAL PROCEDURE}

In this section the experimental duct rig designed for the measurement of the surface pressure distributions over the orifice plate is described. The measured data in this rig will serve to validate the noise model presented in Section II above. The broadband noise PSD predicted from Eq. 14 will be compared with measurements of the sound power radiated from the end of the duct. The procedure for performing the measurements of radiated sound power and duct-wall pressure measurements are briefly described in this section. 
All units: $\mathrm{mm}$

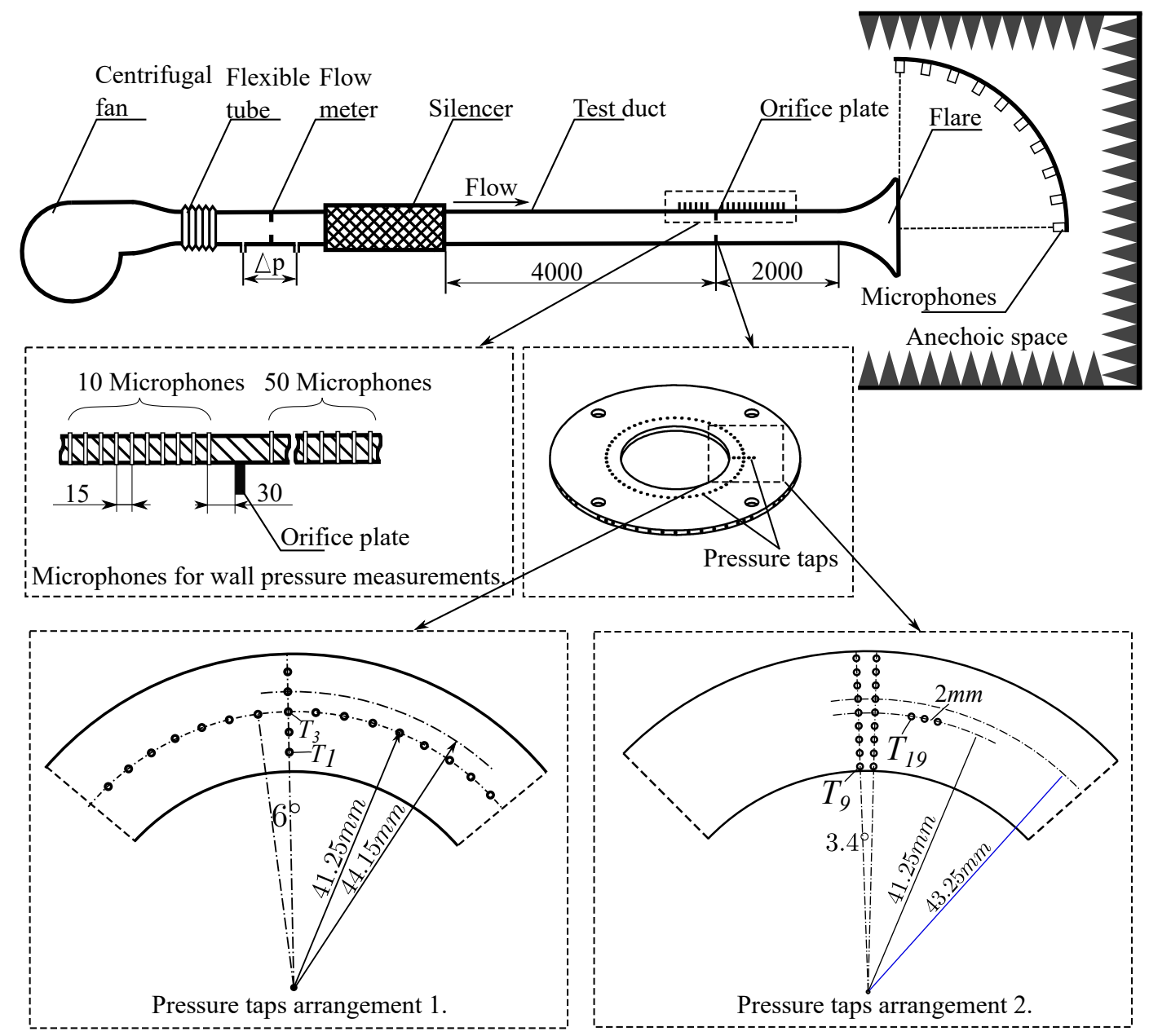

FIG. 3. Schematic of experiment arrangement and the two arrangements of pressure taps on the surface of the orifice plate.

\section{A. Experimental arrangement}

The experimental facility for making the surface pressure and sound power measurements is shown in Fig. 3. The cylindrical duct rig was constructed from acrylic tube with a 0.1 $\mathrm{m}$ internal diameter, and a wall thickness of $5.14 \mathrm{~mm}$. Note that in our rig the duct wall is thicker than in practice and hence the coincidence frequency will be higher than the frequency of interest and hence acoustic structural coupling can be neglected. The duct rig was constructed from a number of smaller acrylic duct sections of varying length connected together with flanges. This arrangement allows the overall length of the duct to be adjusted.

The flow was provided by a centrifugal fan. A flexible tube was installed downstream of the fan to isolate fan vibrations from the rest of the duct. An orifice plate flow meter was 
installed downstream of the flexible tube for the measurements of the mean flow speed in the duct. The distance between the flow meter and the test section was arranged to be long enough to ensure that the flow in the test section was fully developed in the test section. Extraneous noise generated by the fan, the flexible tube and the flow meter, was reduced to levels below the noise due to orifice plate by the use of a silencer located upstream of the test section, as sketched in Fig. 3. To minimise reflections of the sound from the open end back into the duct, a flare was introduced at the end of the duct with a maximum diameter of $0.5 \mathrm{~m}$, corresponding to a cut-off frequency of $186 \mathrm{~Hz}$.

Sound power measurements were made based on acoustic pressure measurements using ten FG-3329-P07 microphones from Knowles Electronics distributed over a $90^{\circ}$ polar arc at a constant distance of $1 \mathrm{~m}$ from the centre of the duct opening, as shown in Fig. 3. The microphone array was located within a small enclosure in which sound absorbing wedges were attached to the wall to provide free field conditions. The wedges have a cut-off frequency of $250 \mathrm{~Hz}$.

The 10 microphones were equally spaced over an angular range of $5^{\circ}<\theta_{n}<85^{\circ}$, in $9^{\circ}$ intervals. The microphones were calibrated using a B\&K 4230 calibrator. Windscreens were attached to the top of the microphones to reduce the effect of flow on the acoustic measurements. Assuming axi-symmetric sound pressure radiation, the sound power can be calculated from

$$
S_{W W}(\omega)=\frac{2 \pi R^{2}}{\rho c_{0}} \sum_{n=1}^{N} S_{p p}\left(\theta_{n}, \omega\right) \sin \theta_{n} \Delta \theta,
$$

where $S_{p p}\left(\theta_{n}, \omega\right)$ is the PSD of sound pressure at $n^{\text {th }}$ measured position $\theta_{n}, R$ is the distance between the centre of the duct open end and the microphone positions, $N=10$ is the total number of microphone, $\Delta \theta$ is the angle interval between two adjacent microphones.

The experimental arrangement required to measure the pressure on the duct wall upstream and downstream of the orifice plate and the surface pressure over the surface of the orifice plate is also shown in Fig. 3. For the duct wall pressure measurement upstream of the orifice plate, a total of 10 microphones were flush-mounted to the duct wall. These were arrange axially along the duct. In the downstream direction a total of 50 microphones were flushed-mounted to the duct wall. The distances between the orifice plate and the first microphones, both upstream and downstream, were $0.03 \mathrm{~m}$. The distance between adjacent 
microphones was $0.015 \mathrm{~m}$. The measured points covered a distance of $0.162 \mathrm{~m}$ upstream of the orifice plate and a distance of $0.765 \mathrm{~m}$ downstream of the orifice plate.

\section{B. Surface pressure measurements}

Measurements of the surface pressure were made using pressure taps 3D printed directed into the orifice plates of $3.3 \mathrm{~mm}$ thickness. The pressure taps were connected to capillary tubes of $1 \mathrm{~mm}$ internal diameter, running inside the orifice plate. Microphones connected along the tube were used to sense the surface pressure fluctuations.

Due the thickness of the orifice plate, the number of pressure taps that can be located on the surface of the orifice plate was limited. To provide maximum coverage of the surface pressure distribution for use in Eqs. 14 and 15 for the radiated acoustic pressure calculation, two arrangements of pressure taps were designed, as shown in Fig. 3.

For the first arrangement, 5 pressure taps were distributed radially at a separation distance of $2.9 \mathrm{~mm}$ and 60 pressure taps separated every $4.3 \mathrm{~mm}$ located circumferentially at the radius of $41.25 \mathrm{~mm}$. For this arrangement it is assumed that the pressure fluctuations over the orifice plate are axi-symmetric and can therefore be completely determined from only a single line of radial pressure taps. The purpose of the circumferential distribution of pressure taps was to provide information about the coherence along this direction.

For the second arrangement, two lines of nine pressure taps along the radial direction were used. The distance between two consecutive taps along the radial direction was $2 \mathrm{~mm}$. The angular separation distance between the two radial lines was $3.4^{\circ}$. The purpose of this arrangement was to provide information about the radial variation in coherence along the radial direction between two adjacent points.

Note that surface pressure information on both sides of the orifice plate was obtained by repeating the measurement with the pressure taps facing the opposite direction.

The purpose of the pressure taps is to provide information about the pressure cross spectra over the surface of the orifice plate. It is therefore essential that the pressure taps, including the tubing and microphones, are accurately calibrated for magnitude and phase. A schematic and photograph of the experimental arrangement for the calibration of the pressure taps is shown in Figs. 4(a) and 4(b) respectively. The calibration of the pressure tap and remote microphone combination was made relative to a reference calibrated microphone. 
As shown in Fig. 4(a), calibration was performed using a loudspeaker installed at the end of a closed horn with a reference microphone located at the other end to measure the acoustic pressure at the surface of the pressure tap. The transfer function was then measured between the pressure at the reference microphone and the remote microphone which was mounted in a T-junction attached roughly $20 \mathrm{~cm}$ along the end of the capillary tube of $1 \mathrm{~mm}$ internal radius, as sketched in Fig. 4(a). The capillary tube was then extended from the T-junction a further $3 \mathrm{~m}$ to avoid reflections from the open end. The remote microphone was a condenser microphone FG-3329-P07 manufactured by Knowles Electronics and the reference microphone was a G.R.A.S. $1 / 4^{\prime \prime}$ microphone.

(a)

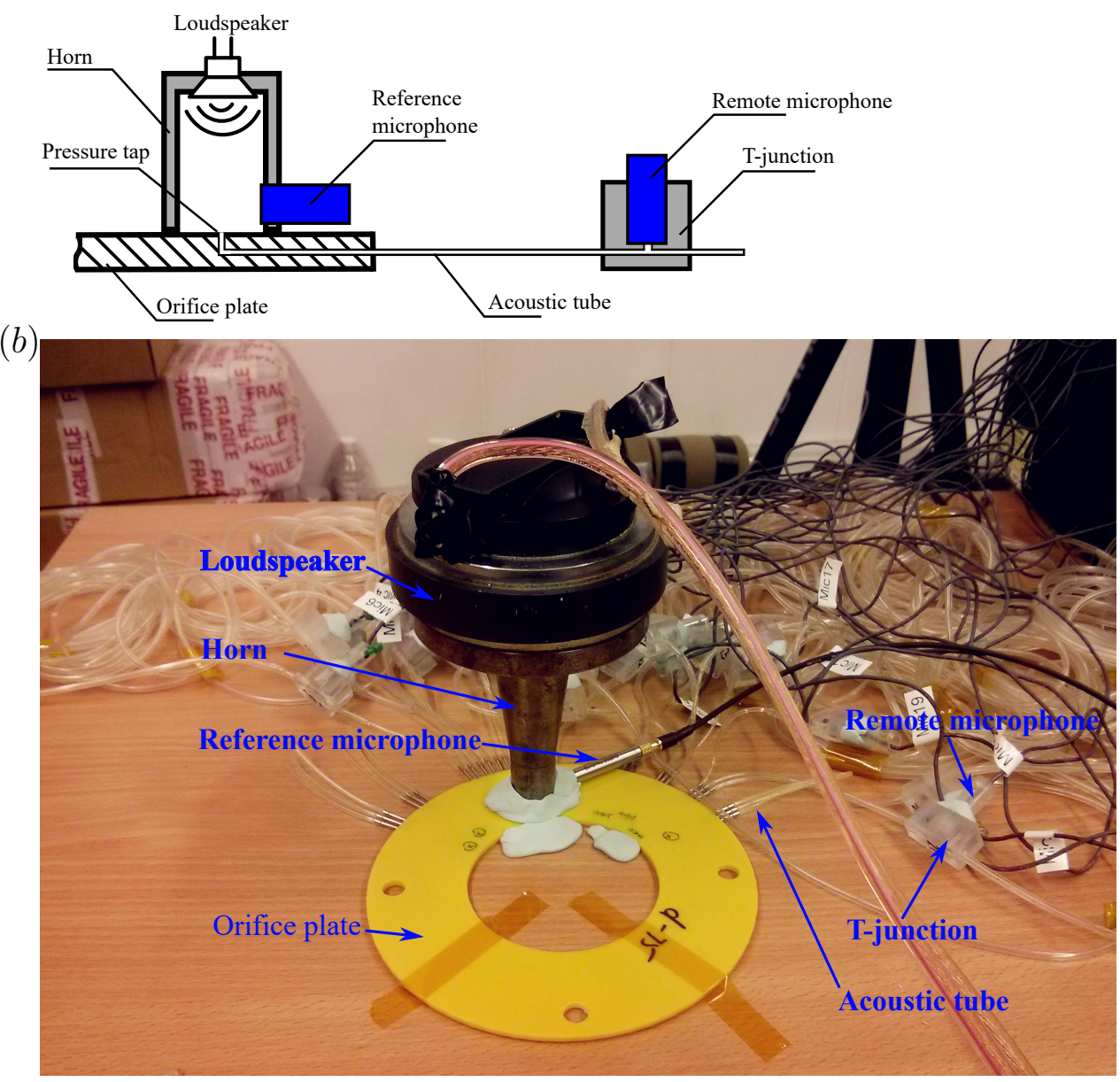

FIG. 4. Experimental arrangement of the calibration of the pressure taps in the orifice plate. (a) Schematic illustration of the calibration of the pressure taps. (b) Photo of the calibration of the pressure taps. 


\section{EXPERIMENTAL RESULTS AND DISCUSSION}

\section{A. Properties of the duct wall pressure and the orifice plate surface pressure}

Maps of the pressure PSD along the duct wall, as a function of axial distance $z$ (normalized by the duct diameter $D$ ) upstream and downstream of the orifice plate with an internal diameter of $65 \mathrm{~mm}$ at a mean flow speed of $10.8 \mathrm{~m} / \mathrm{s}$, is shown in Fig. 5. Downstream positions are denoted by region IV. Negative values of $z / D$ represent locations upstream of the orifice plate, denoted by region I. Also shown in this figure for comparison is the measured pressure on the upstream and downstream surfaces of the orifice plate at a radial position of $47.25 \mathrm{~mm}$, indicated as regions II and III respectively.

The wall pressure spectra upstream of the orifice plate are observed to be typically $20 \mathrm{~dB}$ lower than the highest values in the downstream direction, which occur at $z / D \approx 1.2$. At all upstream locations the pressure spectra is slowly varying and relatively low (approximately $65 \mathrm{~dB})$, suggesting that the flow in this region is relatively smooth. The surface pressure on the upstream side (region II) of the orifice plate is higher by about $10 \mathrm{~dB}$ than on the downstream side (region III). The behaviour of surface pressure spectra of the orifice plate is explored in greater detail below.

Downstream of the orifice plate the highest pressure fluctuations occur at about 1.2 duct diameters of the orifice plate, which is likely to represent the reattachment point as discussed previously. Further downstream of this position, the wall pressure spectra decrease gradually as the distance from the orifice plate increases. Note that the frequency spectrum variation observed in Figure 5 is most likely to be specific to our choice of duct and orifice plate geometric parameters and no universal behaviour can be identified. Nevertheless these results encapsulate the general behaviour of unsteady flow and associated pressure spectrum.

Maps of the upstream surface pressure PSD on the orifice plate versus radial positions (normalized by the duct radius) is shown in Fig. 6(a). Highest pressure fluctuations on the orifice plate are seen to occur near the inner edge, $r_{s} / a=0.65$ (where $r_{s}$ is the radial position of a point on the surface of the orifice plate and $a$ is the internal radius of the duct), and then reduce quite substantially towards the duct wall. Also observed in the regions where the hydrodynamic pressure is relatively low, (i.e, close to the inner duct wall and at frequencies greater than about $4 \mathrm{kHz}$ ), are narrow band peaks corresponding to the duct 


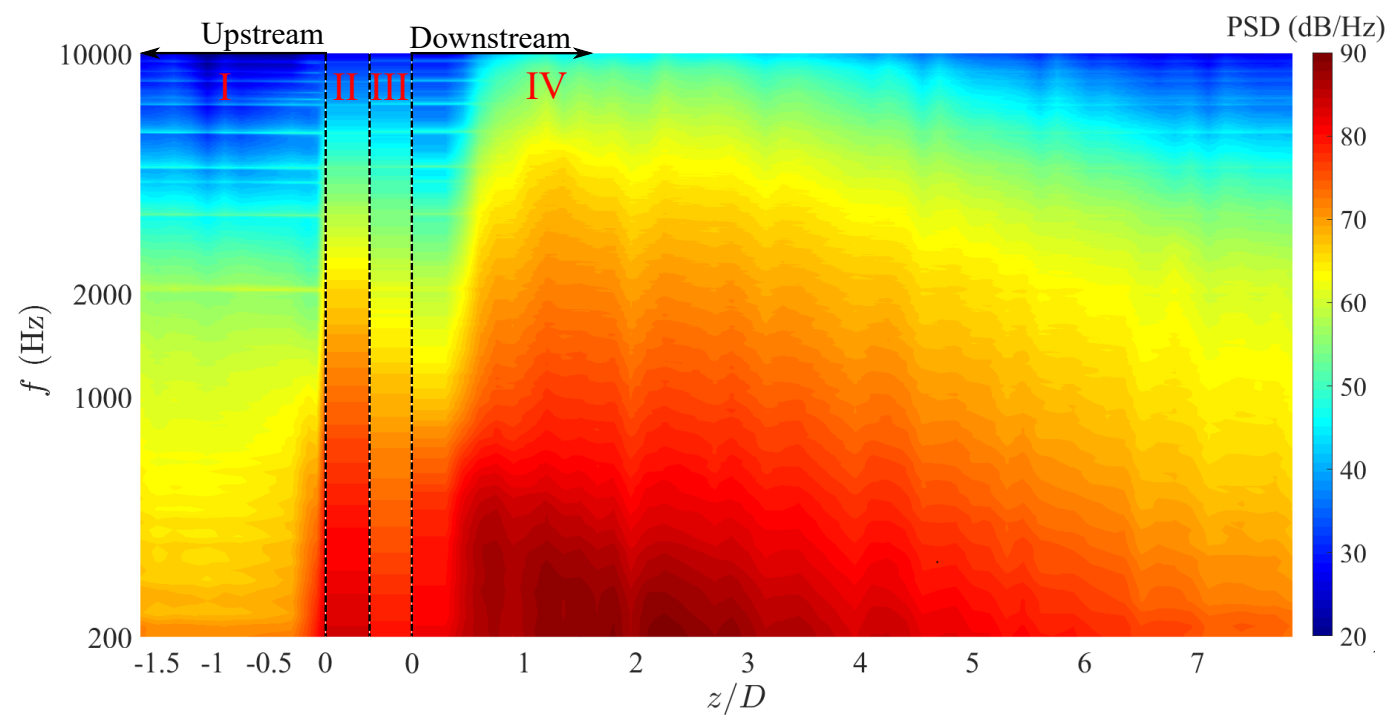

FIG. 5. PSD of the pressure fluctuations on the orifice plate surface and on the duct wall at measured position $z$, region II and region III represent upstream and downstream surface of the orifice plate and region I and region IV represent upstream and downstream duct wall near the orifice plate. The test case has an internal diameter of the orifice plate of $d=65 \mathrm{~mm}$ and a mean flow speed of $U=10.8 \mathrm{~m} / \mathrm{s}$ in the duct.

mode cut-on frequencies.

Maps of the surface pressure PSD versus radial positions (normalized by the radius of the duct) on the downstream surface of the orifice plate is shown in Fig. 6(b). Similar to the upstream side, highest pressure fluctuations also occur at the inner edge of the orifice plate. At radii between the inner edge to about $r_{s} / a=0.75$, the pressure fluctuations decrease gradually. However, at radii greater than $r_{s} / a=0.75$, the surface pressure fluctuations decrease more slowly towards the duct wall.

Fig. 6 clearly demonstrates that pressure fluctuations over the upstream side of the orifice plate are typically $10 \mathrm{~dB}$ greater than those on the downstream side. However, as discussed in Section II B above, the noise generation is also determined by the coherence of the surface pressure. The properties of the coherence are discussed below.

Note also in Fig. 5 that hydrodynamic pressure fluctuations on the duct wall downstream of the orifice plate where the flow reattaches is significantly greater than on the surfaces of the orifice plate, shown as regions II and III in this figure. However, it is straightforward to show that the dipole sources associated with the duct wall pressure fluctuations do not radiate sound efficiently since they are located at the duct wall where the particle velocity normal to the wall is zero. This may be demonstrated formally since the orientation of the 
dipole sources at the duct wall are in the radial direction and at the duct wall the hard-walled boundary condition gives

$$
\frac{\partial G\left(\mathbf{x}, t \mid \mathbf{x}_{s}, \tau\right)}{\partial r_{s}}=0 .
$$

Substituting (19) into Eq. (6) and noting that $\mathbf{f}\left(\mathbf{x}_{s}, \tau\right)$ only has a radial component, gives

$$
p(\mathbf{x}, t)=0,
$$

suggesting that the surface pressure fluctuations on the duct wall do not radiate sound. The dominant noise source is therefore located on the surface of the orifice plate rather than the duct wall where highest pressure fluctuations occur.

The axi-symmetry of the surface pressure fluctuations of the orifice plate is investigated based on the data obtained from pressure tap arrangement 1 sketched in Fig. 3. Fig. 7 shows a comparison of the surface pressure PSD at locations separated circumferentially at a fixed radius of $47.25 \mathrm{~mm}$. Deviations in the spectra are observed to be less than $1 \mathrm{~dB}$ suggesting that the upstream flow behaviour around the orifice plate is strongly axi-symmetric. Similar results are obtained for the downstream surface pressure.

To calculate the sound field in the duct using Eqs. 14 and 15, the cross spectrum must be calculated or measured between every point and every other point on the surface of the orifice plate.

We first investigate the coherence between two points separated radially along the upstream side of the orifice plate. This is plotted in Fig. 8(a) for 5 radial separation distances between $2 \mathrm{~mm}$ and $12 \mathrm{~mm}$ for a mean flow speed of $10.8 \mathrm{~m} / \mathrm{s}$. Along the radial direction, the surface pressure can be seen to have coherence values greater than 0.5 for measurement points separated by $2 \mathrm{~mm}$ at all frequencies up to about $2000 \mathrm{~Hz}$. Between $2000 \mathrm{~Hz}$ and $5000 \mathrm{~Hz}$, the coherence drops to levels greater than about 0.1 . Above $5000 \mathrm{~Hz}$, the coherence drops to near zero. At twice this separation distance of $4 \mathrm{~mm}$, the surface pressure only has significant levels of coherence $(>0.1)$ in the plane wave frequency range lower than about $2000 \mathrm{~Hz}$. At larger separation distances the coherence progressively decreases with increasing separation distance and frequency. For separation distances greater than $8 \mathrm{~mm}$, the coherence falls to nearly 0 .

The coherence at two circumferential separation distances of $2 \mathrm{~mm}$ and $4 \mathrm{~mm}$ is shown 
$(a)$

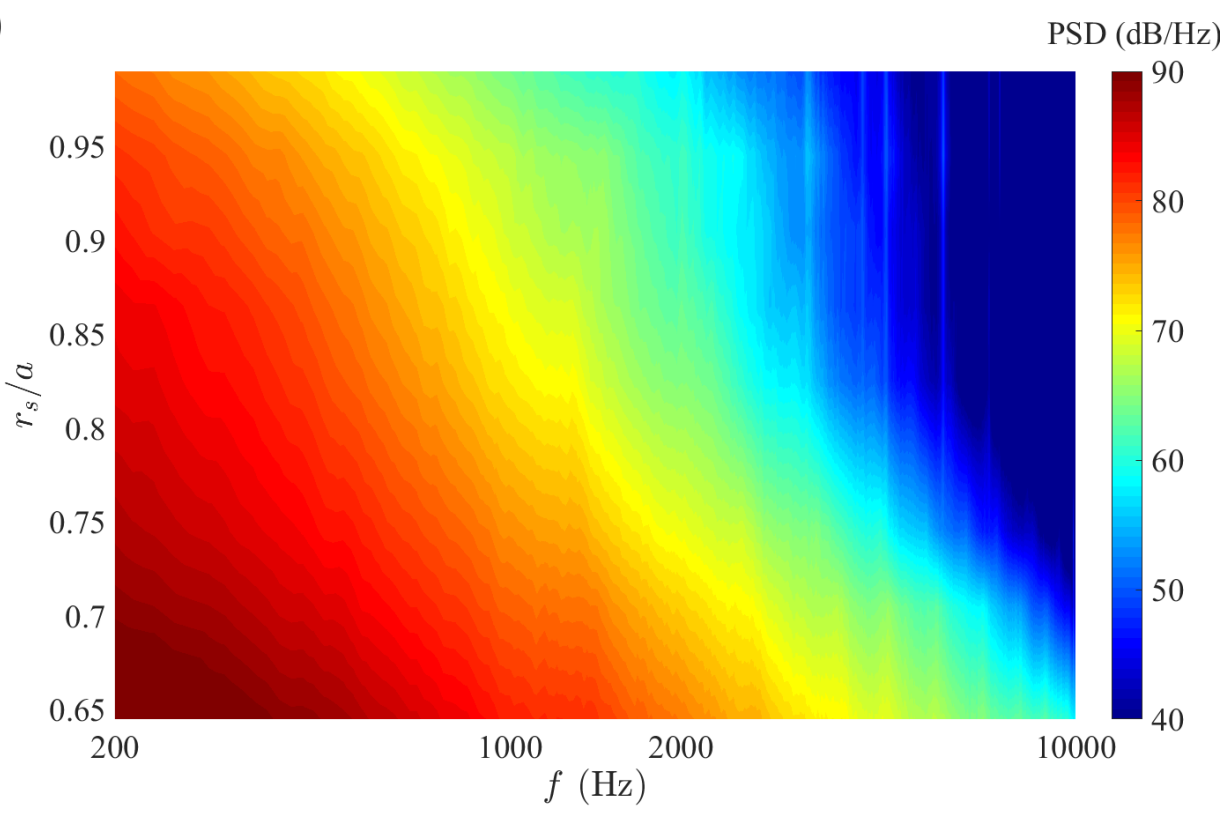

$(b)$

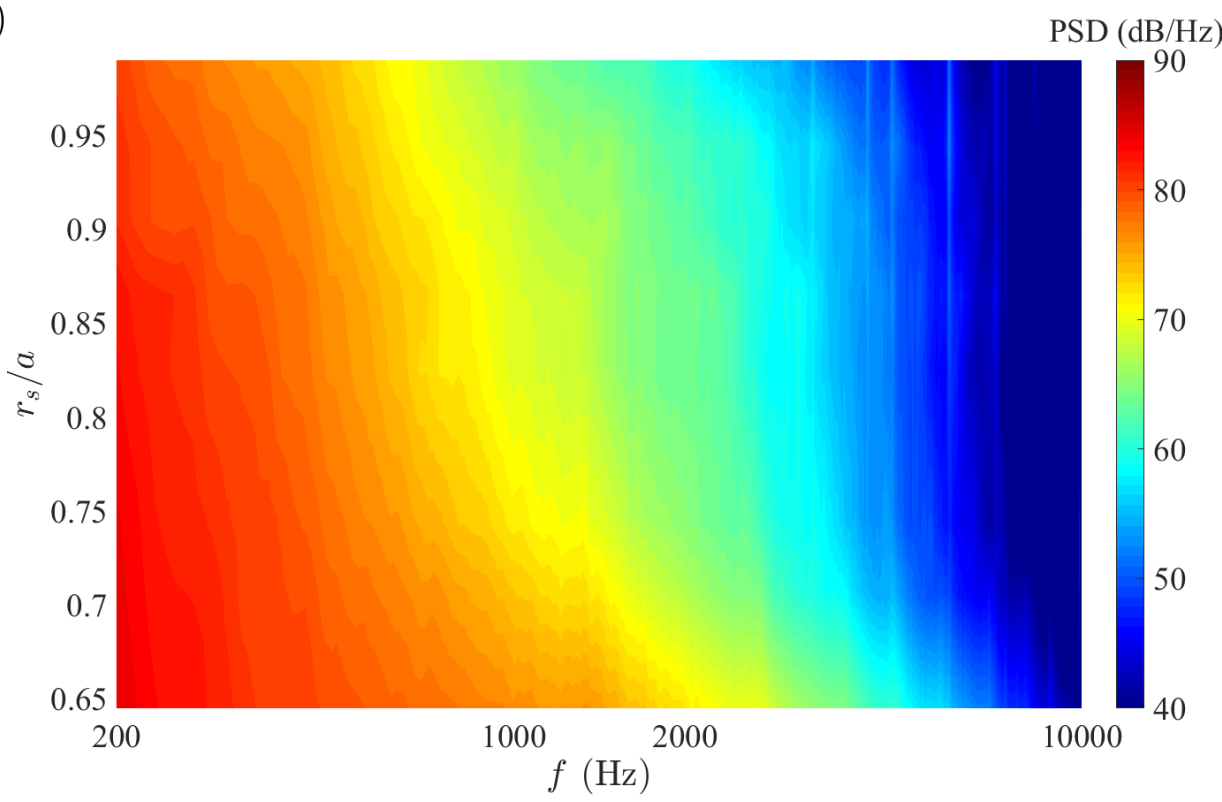

FIG. 6. PSD of the pressure fluctuations on the upstream and downstream surface of the orifice plate along radial direction with an internal diameter of the orifice plate of $d=65 \mathrm{~mm}$ and a mean flow speed of $U=10.8 \mathrm{~m} / \mathrm{s}$ in the duct. (a) PSD of the pressure fluctuation on the upstream surface of the orifice plate. (b) PSD of the pressure fluctuation on the downstream surface of the orifice plate.

in Fig. 8(b) at a radius of $41.25 \mathrm{~mm}$. At the smallest circumferential separation distance of $2 \mathrm{~mm}$, the surface pressure coherence drops from about 0.4 at the lowest frequency of interest to almost zero at frequencies range from about $700 \mathrm{~Hz}$ to $3000 \mathrm{~Hz}$. Above $3000 \mathrm{~Hz}$ the coherence increases. At a separation distance of $4 \mathrm{~mm}$, the coherence is practically zero 


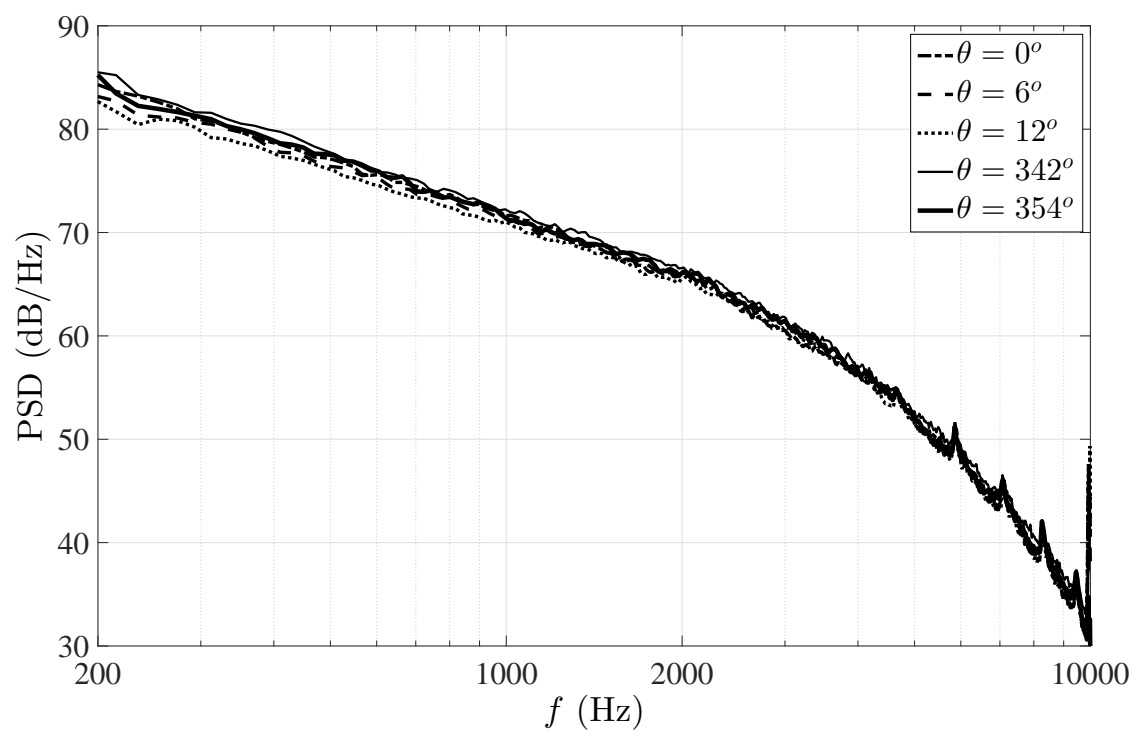

FIG. 7. Comparison of the PSD of the pressure fluctuation on the upstream surface of the orifice plate at a radius of $41.25 \mathrm{~mm}$ along different angular position $\theta$ with an internal diameter of the orifice plate of $d=65 \mathrm{~mm}$ and a mean flow speed of $U=10.8 \mathrm{~m} / \mathrm{s}$ in the duct.

until about $3000 \mathrm{~Hz}$, above which almost identical coherence values are observed as for the $2 \mathrm{~mm}$ separation distance. Above $3000 \mathrm{~Hz}$, the presence of the cut-on frequencies is clearly observed in the coherence spectra suggesting that in this frequency range the pressure on the surface of the orifice plate is dominated by the acoustic field.

Fig. 9 shows the surface pressure coherence on the downstream side of the orifice plate, which can be observed to have similar behaviour to that of the upstream surface, but slightly higher in level.

The magnitude of the cross spectral density (CPSD) of the pressure fluctuation on the upstream surface of the orifice plate along the radial and circumferential directions is shown in Fig. 10. The separation distances plotted in this figure correspond to the coherence measurements shown in Fig. 8. Consistent with the coherence, the magnitude of the CPSD drops rapidly as the separation distance increases. Along the radial direction, the magnitude of the CPSD for separation distances of $6 \mathrm{~mm}$ and above are more than 10dB below the PSD of the surface pressure at the reference position. As the separation distance increases above $6 \mathrm{~mm}$ the cross spectrum ceases to change, consistent with near-zero values of the coherence function. Along the circumferential direction, similar behaviour of the CPSD is observed, where at separation distances above $3 \mathrm{~mm}$, the magnitude of the CPSD ceases to 
(a)

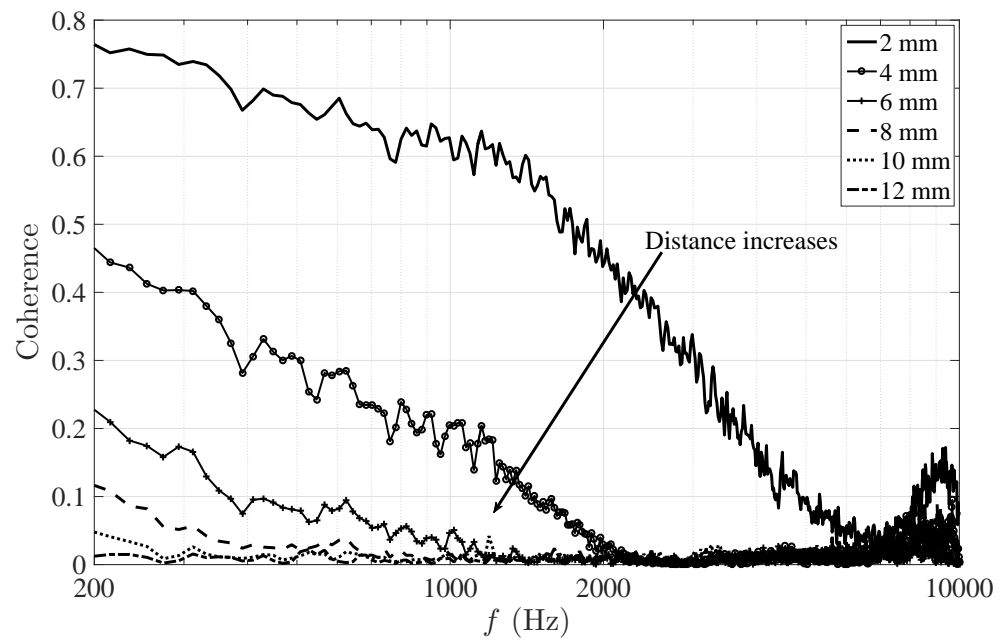

(b)

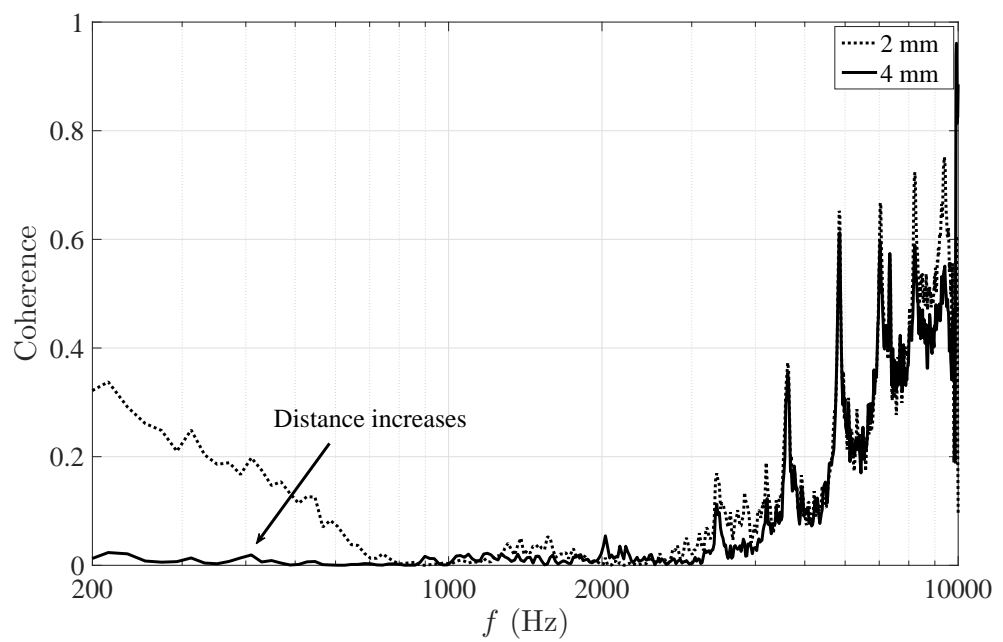

FIG. 8. Coherence of the surface pressure with different separation distance on the upstream side of the orifice plate with an internal diameter of the orifice plate of $d=65 \mathrm{~mm}$ and a mean flow speed of $U=10.8 \mathrm{~m} / \mathrm{s}$ in the duct. For radial direction, the coherence was measured between pressure tap $T_{9}$ and other pressure taps in radial direction. For circumferential direction, the coherence was between pressure tap $T_{19}$ and other pressure taps along circumferential direction. (a) Coherence of the surface pressure on the upstream side along radial direction. (b) Coherence of the surface pressure on the upstream side along circumferential direction.

Based on the observations discussed above, a summary of the characteristics of the surface pressure on the orifice plate is given below:

- The surface pressure is axi-symmetric to significantly less than $1 \mathrm{~dB}$.

- The PSD of the surface pressure on the upstream side is higher than on the downstream 
(a)

(b)
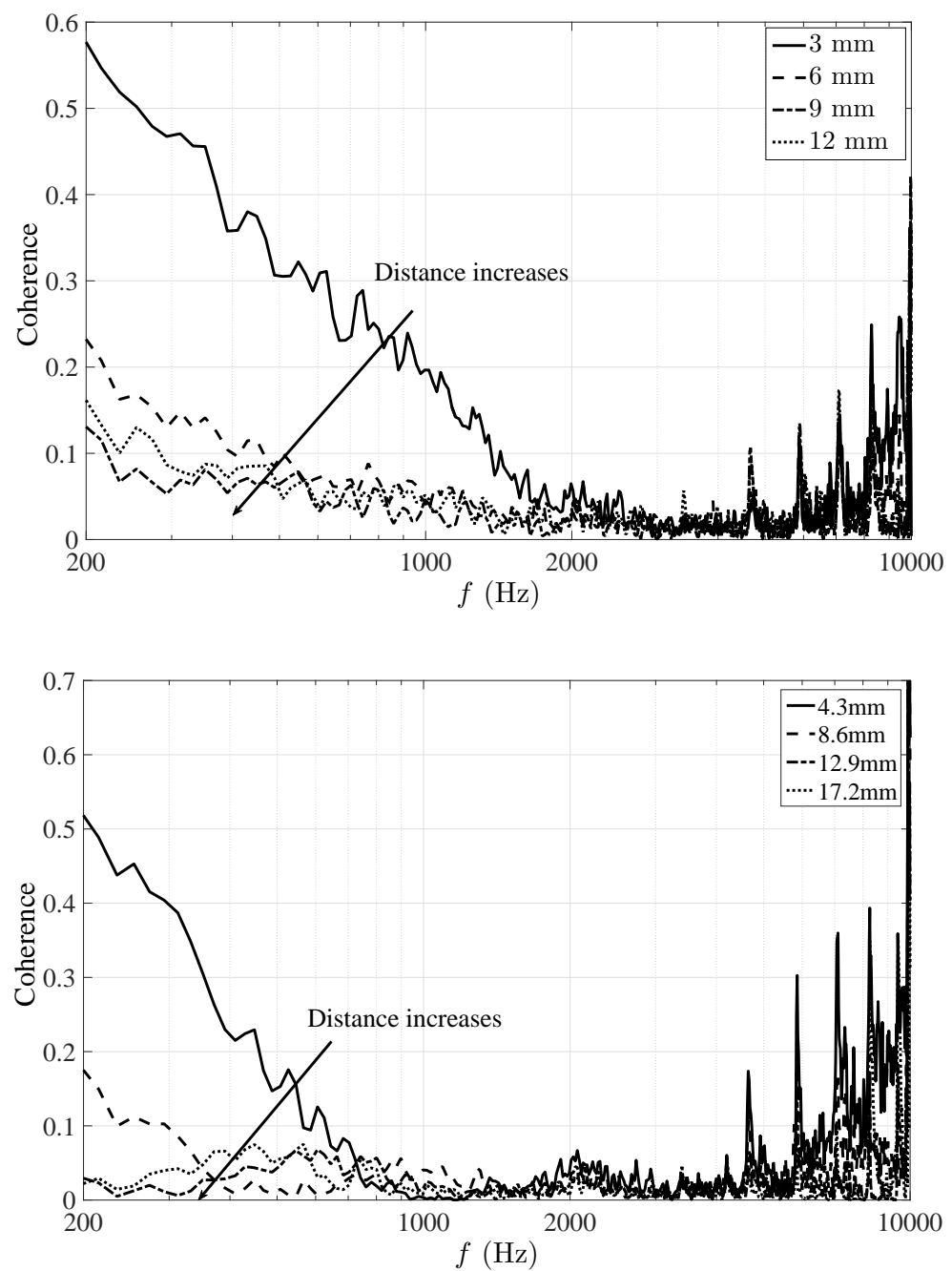

FIG. 9. Coherence of the surface pressure with different separation distance on the downstream side of the orifice plate with an internal diameter of the orifice plate of $d=65 \mathrm{~mm}$ and a mean flow speed of $U=10.8 \mathrm{~m} / \mathrm{s}$ in the duct. For radial direction, the coherence was between pressure tap $T_{1}$ and other pressure taps in radial direction. For circumferential direction, the coherence was between pressure tap $T_{3}$ and other pressure taps along circumferential direction. (a) Coherence of the surface pressure on the downstream side along radial direction. (b) Coherence of the surface pressure on the downstream side along circumferential direction.

side. On both sides, the highest pressure fluctuation occurs at the inner edge of the orifice plate.

- On both sides, the surface pressure is only coherent within a relatively small distance of several millimetres. In the plane wave frequency range, the coherence length (defined here as when the coherence is greater than 0.1 ) is about $4 \mathrm{~mm}$ in the radial direction and $1.5 \mathrm{~mm}$ in the circumferential direction at a mean flow speed of $10.8 \mathrm{~m} / \mathrm{s}$. Above 
(a)

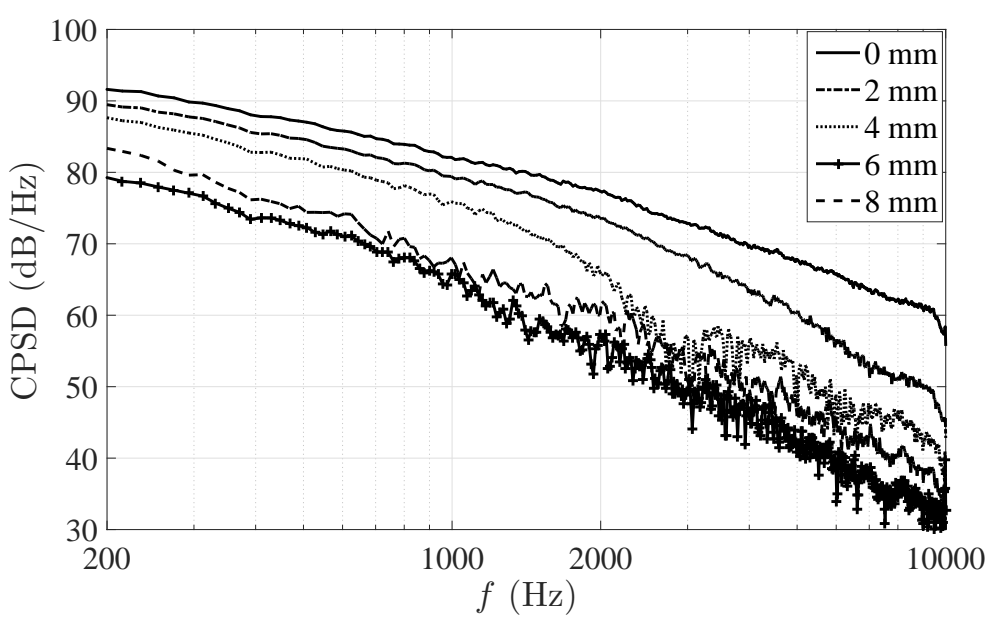

$(b)$

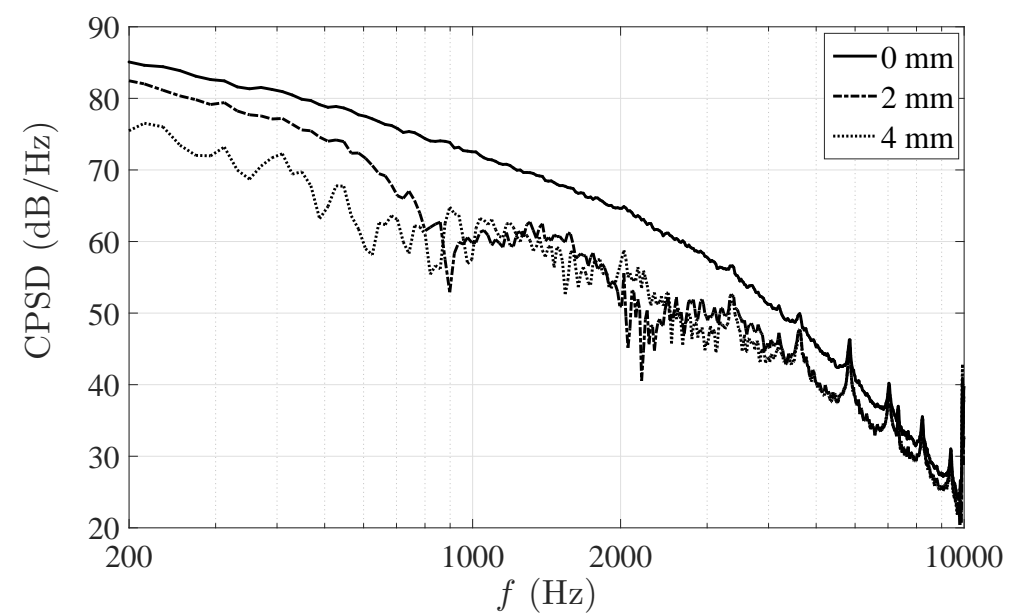

FIG. 10. CPSD of the pressure fluctuation on the upstream surface of the orifice plate along radial direction and circumferential direction with an internal diameter of the orifice plate of $d=65 \mathrm{~mm}$ and a mean flow speed of $U=10.8 \mathrm{~m} / \mathrm{s}$ in the duct. (a) Cross spectral density (CPSD) of the pressure fluctuation on the upstream surface of the orifice plate along radial direction. (b) Cross spectral density (CPSD) of the pressure fluctuation on the upstream surface of the orifice plate along circumferential direction.

the first cut-on frequency, the coherence length is about $2 \mathrm{~mm}$ in the radial direction and less than $1.5 \mathrm{~mm}$ in the circumferential direction. The coherence length is therefore larger in the radial direction than in the circumferential direction, and larger on the downstream side than on the upstream side. 


\section{B. Sound field prediction in the duct based on the surface pressure}

The use of Eqs. 14 and 15 to calculate the sound field in the duct induced by the orifice plate requires information about the surface pressure cross spectra over the entire surface. However, owing the thickness of the orifice plate, the number of pressure taps that can be located into the plate is limited. The CPSD between any two points on the surface of the orifice plate is therefore approximated using the limited surface pressure measurements collected from the arrangement of pressure taps sketched in Fig. 3 and the properties of the surface pressure listed above.

A schematic illustration of the method to estimate the CPSD at all combinations of points is shown in Fig. 11. The CPSD between any two points along the radial direction can be calculated based on the arrangement in Fig. 3. As the surface pressure is axi-symmetric, the measured CPSD along the radial direction therefore applies to all circumferential angles $\theta$. The CPSD between two points at different radial locations is approximated by the CPSD between the two points separated by the closest distance. However, these closest positions are not in the radial direction due to the CPSD along the radial direction being much higher than between two points at different radial positions, as shown in Fig. 11.

For example, consider the calculation of the CPSD between point $T_{4}$ and an arbitrary point $T_{i}$. The CPSD between pressure tap $T_{3}$ and the pressure taps along the circumferential direction are first measured. A series of cross spectral values with different separation distances are therefore obtained. The CPSD between points $T_{3}$ and $T_{i}$ is then assumed to be identical to the CPSD between two points with closest separation distance. This approximation is accurate for three reasons. First, the variation of the CPSD of the surface pressure along the circumferential direction is small. Second, the CPSD tend to a constant level as separation distance increases, which is most likely due to the acoustic pressure contribution to the total pressure, which is coherent over a much larger distance than the hydrodynamic contribution. Third, the coherence between the pressure taps near the inner edge is higher than that near the duct wall and therefore the CPSD between the pressure taps along the middle line of the orifice plate is approximated by the average coherence of the two sides. Using these approximations the CPSD between any two points can be estimated. However, in practice only the CPSD between two points within an area over which the surface pressure is significantly coherent $(>0.1)$ will contribute significantly to 


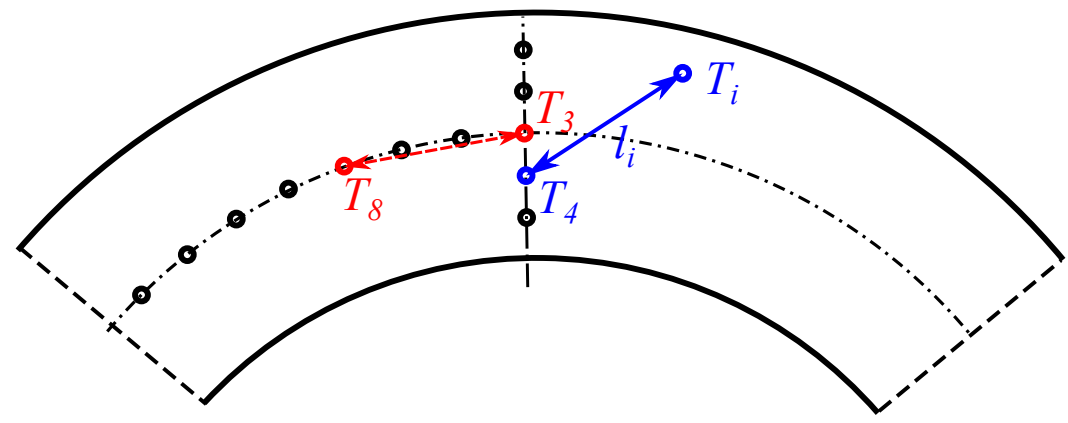

FIG. 11. Illustration of the approximate calculation of the surface pressure cross spectrum density between any two points.

Eqs. 14 and 15 for the radiated acoustic pressure. This coherence area will be calculated in the next section.

In the plane wave frequency range, the PSD of sound power $S_{W W}(\omega)$ can be estimated by assuming reflection from the open end can be neglected, in which case,

$$
S_{W W}(\omega)=\frac{\pi a^{2}}{\rho c} S_{p p}(\mathbf{x}, \omega),
$$

where $S_{p p}(\mathbf{x}, \omega)$ is the acoustic pressure at any point in the duct $\mathbf{x}$, which in the plane wave frequency range is independent of $\mathbf{x}$. The sound power calculated from Eq. 21 can be compared with the sound power measured directly from Eq. 18 by integration of the far field sound intensity. A representative comparison of the sound power spectra obtained at different flow speeds is shown in Fig. 12. The solid curves represent the sound power obtained from the far-field acoustic measurements and the dash curves represent the sound power estimate based on Eq. 14 using the surface pressure measurements. The agreement between two spectra is better than $2 \mathrm{~dB}$ at frequencies above about $300 \mathrm{~Hz}$ at flow speeds below $17.5 \mathrm{~m} / \mathrm{s}$. At higher flow speeds, $20.0 \mathrm{~m} / \mathrm{s}$ and $25.0 \mathrm{~m} / \mathrm{s}$, agreement is poorer below $1000 \mathrm{~Hz}$. The reason for this discrepancy is currently unclear.

\section{Surface pressure coherence area}

The measured coherence results in Figs. 8 and 9 indicate that at any particular frequency, the surface pressure is only coherent over a relatively small area, which we shall refer to as the coherence area. If it is assumed that the surface pressure PSD remains roughly constant within the coherence area (since its dimensions are much smaller than the acoustic and 




FIG. 12. The comparison of PWL spectra from far field measurements and surface pressure measurements for the orifice plate with an internal diameter of $d=65 \mathrm{~mm}$ under different mean flow speeds in the duct.

hydrodynamic wavelengths), the cross spectrum integrated over the surface required by Eq. 12 can be approximated by

$$
\int_{s^{\prime}} S_{f f}\left(\mathbf{x}_{s}, \mathbf{x}_{s}^{\prime}, \omega\right) d S\left(\mathbf{x}_{s}^{\prime}\right)=S_{f f}\left(\mathbf{x}_{s}, \omega\right) A_{c}\left(\mathbf{x}_{s}, \omega\right)
$$

where $A_{c}\left(\mathbf{x}_{s}, \omega\right)$ is the coherence area, and is defined here as the area centred on $\mathbf{x}_{s}$ at frequency omega at which all pairs of points within it have coherence values greater than 0.1. Substituting Eq. 22 into Eq. 14 and noting from Eq. 16 that $S_{f f}\left(\mathbf{x}_{s}, \mathbf{x}_{s}^{\prime}, \omega\right)=$ $S_{p p}^{+}\left(\mathbf{x}_{s}, \mathbf{x}_{s}{ }^{\prime}, \omega\right)+S_{p p}^{-}\left(\mathbf{x}_{s}, \mathbf{x}_{s}{ }^{\prime}, \omega\right)$ yields the following relationship between the PSD of the noise averaged over a duct cross section, and $\left\langle S_{p p}^{+}(\omega)\right\rangle_{S r}$ and $\left\langle S_{p p}^{-}(\omega)\right\rangle_{S r}$, the surface pressure PSD averaged over the upstream and downstream surfaces of the orifice plate respectively,

$$
\left\langle A_{c}(\omega)\right\rangle_{S r}=\frac{\left\langle S_{p p}(\omega)\right\rangle_{S}}{\left\langle S_{p p}^{-}(\omega)\right\rangle_{S r}+\left\langle S_{p p}^{+}(\omega)\right\rangle_{S r}} \frac{4 A^{2}}{A_{S_{r}}} .
$$

where $\langle\ldots\rangle_{S}$ refers to quantities averaged over the duct cross sectional areas, such that $\left\langle A_{c}(\omega)\right\rangle_{S r}$ denotes the coherence area averaged over the orifice plate surface, and $A_{S_{r}}$ is the surface area of the orifice plate. 


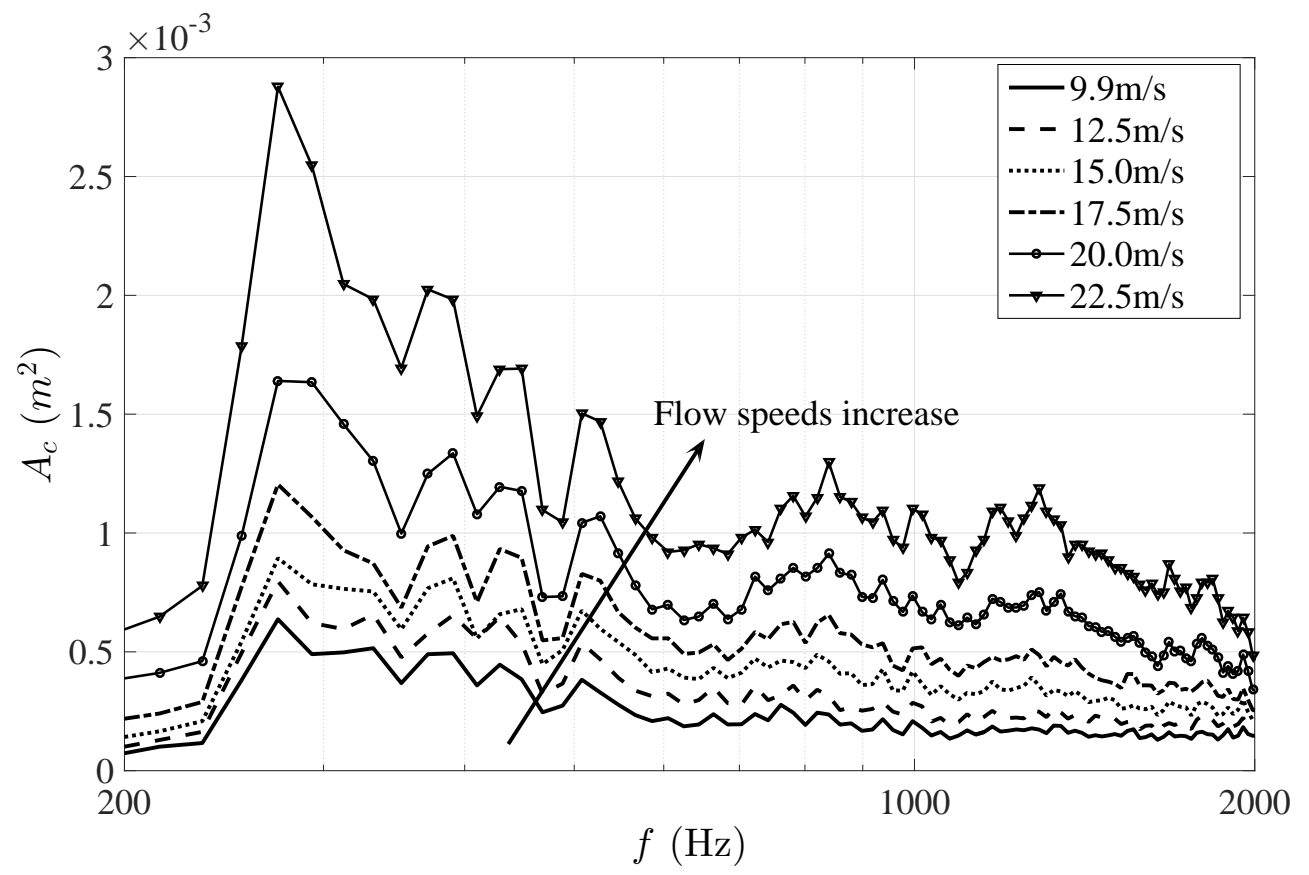

FIG. 13. The average coherence area in plane wave frequency range for the orifice plate with an internal diameter of $d=65 \mathrm{~mm}$ under different mean flow speeds in the duct.

The average coherence area calculated from Eq. 23 for different flow speeds are plotted in Fig. 13 against frequency. The coherence length may be approximately calculated from $l_{c}(\omega) \propto \sqrt{\left\langle A_{c}(\omega)\right\rangle}$ and is about $1.6 \mathrm{~cm}$ at $1000 \mathrm{~Hz}$ at a mean flow speed of $9.9 \mathrm{~m} / \mathrm{s}$.

Nelson and Morfey [13] speculated that the coherence length should vary as $l_{c} \propto U / f$ and hence the correlation area as $A_{c} \propto U^{2} / f^{2}$. In Fig. 14, the sound power, surface pressure and coherence area obtained from Eq. 23 integrated over the plane wave frequency range, are plotted versus flow speed. Based on just the five flow speeds investigated, the sound power in this frequency bandwidth can be seen to obey a $U^{5.7}$ scaling law and the surface pressure a $U^{3.9}$ scaling law. This suggests from Eq. 23 a scaling law for the coherence area of $U^{1.8}$, which is consistent with Nelson and Morfey's speculated dependence of $U^{2}$. However, Fig. 13 shows a weaker frequency dependence than the $f^{-2}$ dependence speculated by Nelson and Morfey.

\section{Sound source distribution on the surface of the orifice plate}

Eqs. 14 and 15 provide the relationship between the source strength CPSD over the surface of the orifice plate and the radiated sound pressure spectral density. Eq. 14 for the 


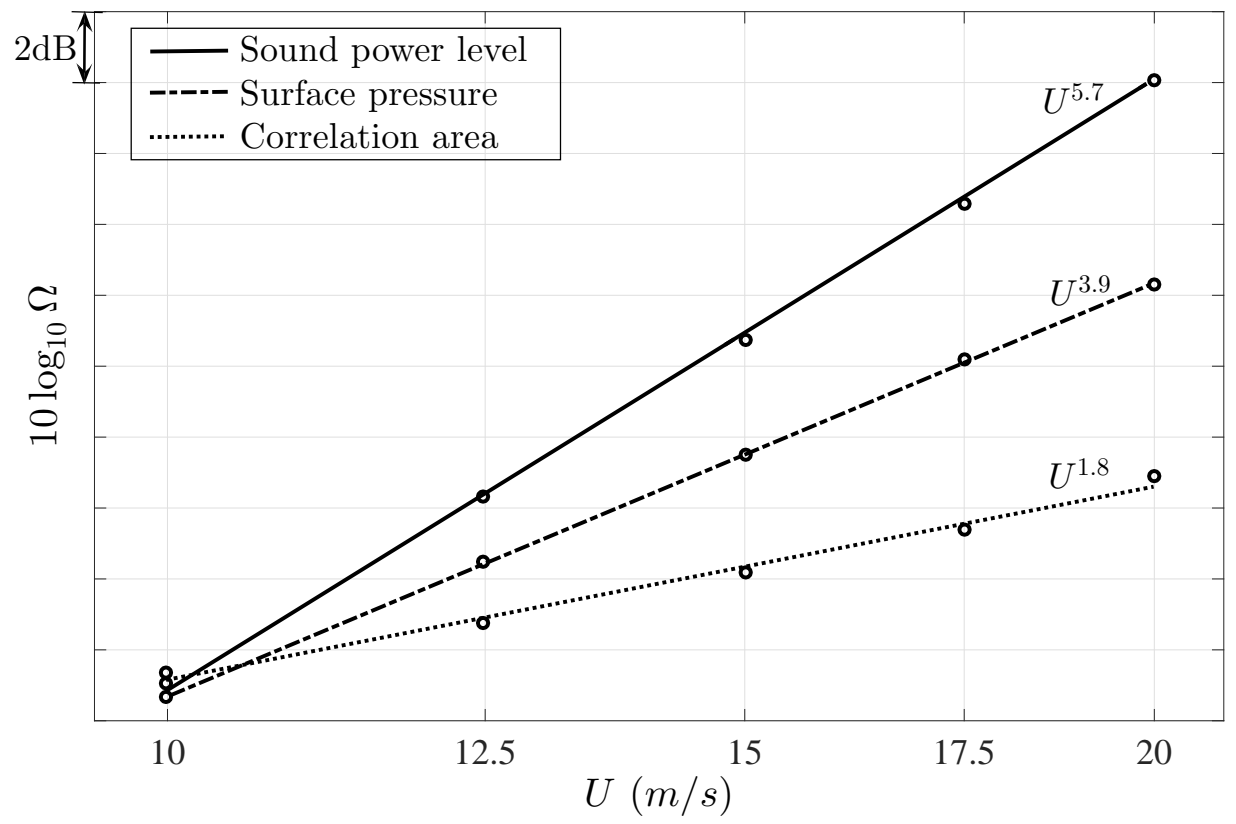

FIG. 14. Different velocity scaling laws for sound power, surface pressure and correlation area, $\Omega$ represents sound power, sound pressure and correlation area integrated over $500-2000 \mathrm{~Hz}$ respectively.

radiated pressure, valid in the plane wave frequency range, can be re-expressed as a source strength distribution $\hat{S}$ that includes the coherence area, integrated over the surface of the orifice plate, i.e.,

$$
S_{p p}(\mathbf{x}, \omega)=\frac{1}{4 A^{2}} \int_{S_{r}} \hat{S}_{f f}\left(\mathbf{x}_{s}, \omega\right) d S\left(\mathbf{x}_{s}\right)
$$

where $\hat{S}_{f f}$ is given by

$$
\hat{S}_{f f}\left(\mathbf{x}_{s}, \omega\right)=\int_{S_{r}^{\prime}} S_{f f}\left(\mathbf{x}_{s}, \mathbf{x}_{s}^{\prime}, \omega\right) d S\left(\mathbf{x}_{s}^{\prime}\right)
$$

In this work we assume that the source term $\hat{S}_{f f}$ is axi-symmetric and that all quantities are slowly varying over the coherence area, in which case,

$$
\hat{S}_{f f}\left(r_{s}, \omega\right)=\int_{S^{\prime}} S_{f f}\left(r_{s}, r_{s}^{\prime}, \omega\right) d S\left(r_{s}^{\prime}\right) \approx S_{f f}\left(r_{s}, \omega\right) A_{c}\left(r_{s}, \omega\right)
$$

Under the assumption of axi-symmetry, Eq. 14 for the radiated pressure spectrum in the plane wave frequency range becomes 

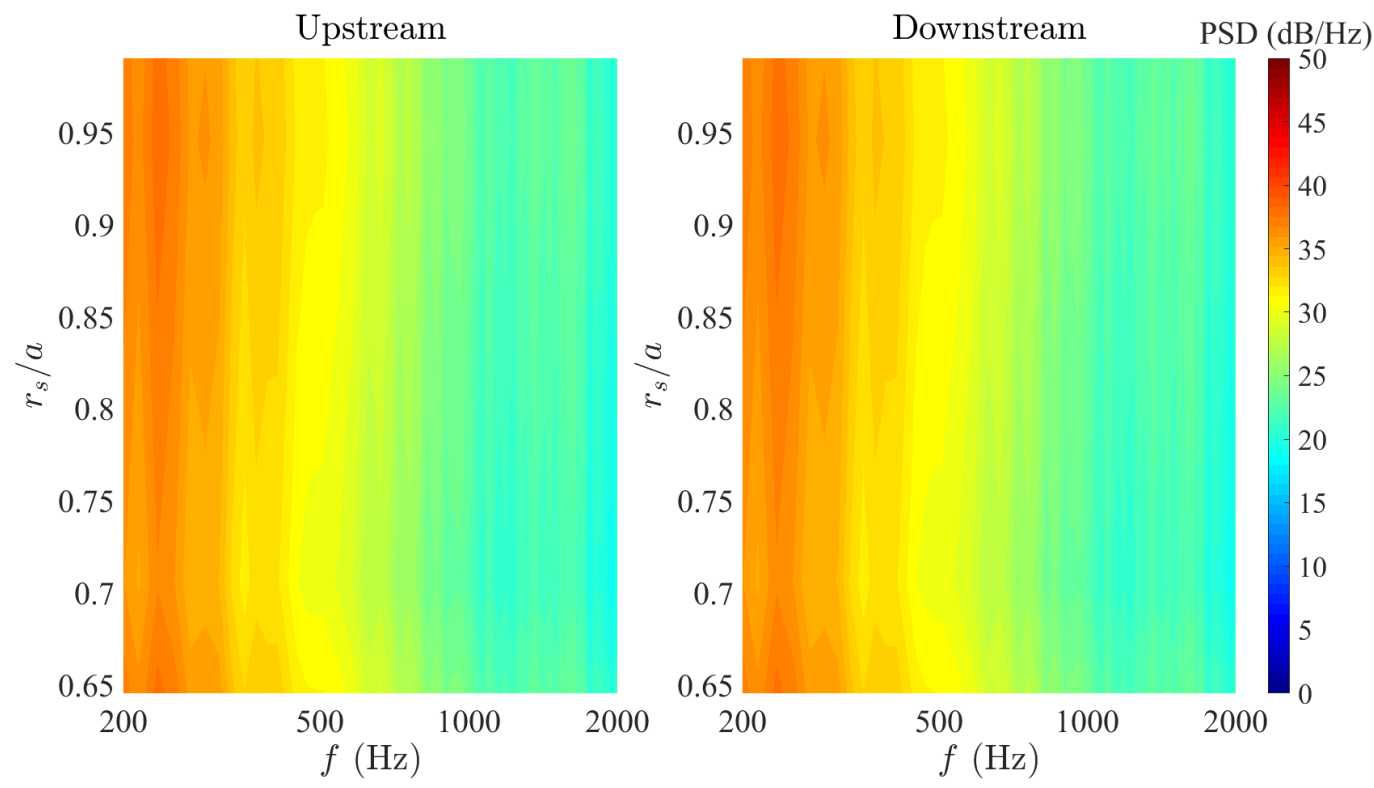

FIG. 15. Sound source distribution on the surface of the orifice plate for the plane wave. The test case is for an internal diameter of $65 \mathrm{~mm}$ under a mean flow speed of $9.9 \mathrm{~m} / \mathrm{s}$.

$$
S_{p p}(\mathbf{x})=2 \pi \int_{r_{1}}^{r_{2}} \hat{S}_{f f}\left(r_{s}, \omega\right) r_{s} d r_{s},
$$

where $r_{1}$ and $r_{2}$ are the inner and outer radii of the orifice plate.

The sound source distribution $\hat{S}_{f f}\left(r_{s}, \omega\right)$ calculated from Eq. 26 over the plane wave frequency range is shown in Figs. 15 over the upstream and downstream sides of the orifice plate respectively. The source distributions $\hat{S}_{f f}\left(r_{s}, \omega\right)$ on the upstream and downstream surfaces of the orifice plate are observed to be similar in both their radial variation and level. Even though the surface pressure on the upstream side is significantly higher on the upstream surface than the downstream surface, as shown in Fig. 6, the coherence on the upstream side is smaller than on the downstream side as shown in Figs. 8 and 9 respectively. The combination of these factors leads that the source distribution on both sides, taking into account the coherence area, being similar on both sides of the orifice plate as shown in Fig. 15.

\section{CONCLUSIONS}

This work presents an experimental investigation into the noise generation due to a single hole orifice plate introduced inside a hard walled circular duct containing a uniform mean 
flow. The relationship between surface pressure fluctuations over the surface of the orifice plate and the in-duct sound field is explored. The surface pressure cross spectra over the orifice plate has been measured to deduce the in-duct sound field generated by the orifice plate. The estimated sound field based on the surface pressure measurements are generally shown to be within about $2 \mathrm{~dB}$ of the measured spectra over a wide range of frequencies and flow speeds. However, largest error of about $5 \mathrm{~dB}$ is observed at the highest flow speed investigated, the reasons for which are currently unclear.

The pressure spectrum on the duct wall and the surface of the orifice plate was measured in this work. It is found that the duct wall pressure upstream of the orifice plate is generally lower compared to the typical pressure on the surface of the orifice plate. Highest duct wall pressures were found to occur around the distance at which the flow reattaches to the duct wall where it is more than $10 \mathrm{~dB}$ higher than typical values on the surface of the orifice plate. However, despite this observation we argue in this work that these duct wall pressure fluctuations are non-radiating and that the noise radiated into the duct is due to the unsteady pressure difference acting across the orifice plate.

Largest surface pressure fluctuations are found to occur at the inner corner of the orifice plate on the upstream side. However, the coherence values between two points on the upstream surface was found to be lower than on the downstream side. The source distribution (per unit area), taking into account the area over which the surface pressure are coherent, was shown to be approximately the same on both sides of the orifice place and fairly uniform along the radius of the orifice plate.

The surface pressure fluctuations were shown to obey a $U^{3.9}$ velocity scaling law, while the sound power transmitted along the duct was found to follow a $U^{5.7}$ velocity scaling law. This implies that the surface pressure coherence area follows a $U^{1.8}$ velocity scaling law, which is very close to the $U^{2}$ scaling law speculated in previous work.

\section{ACKNOWLEDGMENTS}

This work is part of the first author's doctoral thesis. The financial support of Airbus and China Scholarship Council (CSC) is greatly appreciated. 
[1] F. Durst and A. B. Wang, "Experimental and numerical investigations of the axisymmetric, turbulent pipe flow over a wall-mounted thin obstacle," Proceedings of 7th Symposium on Turbulenct Shear Flows. 1, 0.4.1-10.4.6 (1989).

[2] G. H. Nail, "A study of 3-dimensional flow through orifice meters," PhD Thesis, Texas A\& M University, 1991.

[3] S. Feng, F. Atsushi, T. Tatsuya and T. Yoshiyuki, "Particle image velocimetry measurements of flow field behind a circular square-edged orifice in a round pipe," Experiments in Fluids. 54(6), 1-18 (2013).

[4] N. K. Agarwal and M. K. Bull, "Characteristics of the flow separation due to an orifice plate in fully-developed turbulent pipe-flow," Eighth Australasian Fluid Mechanics Conference, 1983.

[5] N. K. Agarwal, "Identification of higher order acoustic modes in distributed pipe flow," Journal of Sound and Vibration. 129(1), 166-167 (1989).

[6] N. K. Agarwal, "Mean separation and reattachment in turbulent pipe flow due to an orifice plate," Journal of Fluids Engineering. 116(2), 373-376 (1994).

[7] N. K. Agarwal, "The sound field In fully developed turbulent pipe flow due to internal flow separation, part II: modal amplitude and cut-off frequencies," Journal of Sound and Vibration. 175(1), 65-76 (1994).

[8] N. K. Agarwal, "The sound field in fully developed turbulent pipe flow due to internal flow separation, part I: wall-pressure fluctuations," Journal of Sound and Vibration. 169(1), 89-109 (1994).

[9] N. K. Agarwal and M. K. Bull, "Acoustic wave propagation in a pipe with fully developed turbulent flow," Journal of Sound and Vibration. 132(2), 275-298 (1989).

[10] E. J. Kerschen and J. P. Johnston, "Mode selective transfer of energy from sound propagating inside circular pipes to pipe wall vibration," The Journal of the Acoustical Society of America. 67(6), 1931-1934 (1980).

[11] E. J. Kerschen and J. P. Johnston, "A modal separation measurement technique for broadband noise propagating inside circular ducts," Journal of Sound and Vibration. 76(4), 499-515 (1981).

[12] E. J. Kerschen and J. P. Johnston, "Modal content of noise generated by a coaxial jet in a 
pipe," Journal of Sound and Vibration. 76(1), 95-115 (1981).

[13] P. A. Nelson and C. L. Morfey, "Aerodynamic sound production in low speed flow ducts," Journal of Sound and Vibration. 79(2), 263-289 (1981).

[14] Gordon, Colin G., "Spoiler generated flow noise. II. results," Journal of the Acoustical Society of America. 45(1), 214-223 (1969).

[15] Gordon, Colin G., "Spoiler generated flow noise. I. the experiment," Journal of the Acoustical Society of America. 43(5), 1041-1048 (1968).

[16] Oldham, D. J. and Ukpoho, A. U., "A pressure-based technique for predicting regenerated noise levels in ventilation systems," Journal of Sound and Vibration. 140(2), 259-272 (1990).

[17] Karekull, Oscar and Efraimsson, Gunilla and Abom, Mats, "Revisiting the NelsonMorfey scaling law for flow noise from duct constrictions," Journal of Sound and Vibration. 357(2), 233-244 (2015).

[18] N. Curle, "The influence of solid boundaries upon aerodynamic sound," Proceedings of the Royal Society of London. Series A, Mathematical and Physical Sciences. 231(1187), 505-514 (1955).

[19] M. Goldstein, "Aeroacoustics," McGraw-Hill Press, New York. (1976).

Fig. 1. Different flow regions in a duct with an orifice plate [8].

Fig. 2. Illustration of an orifice plate in an infinite cylinder and cylindrical coordinates system used for theoretical developments.

Fig. 3. Schematic of experiment arrangement and the two arrangements of pressure taps on the surface of the orifice plate.

Fig. 4. Experimental arrangement of the calibration of the pressure taps in the orifice plate. (a) Schematic illustration of the calibration of the pressure taps. (b) Photo of the calibration of the pressure taps.

Fig. 5. PSD of the pressure fluctuations on the orifice plate surface and on the duct wall at measured position $z$, region II and region III represent upstream and downstream surface of the orifice plate and region I and region IV represent upstream and downstream duct wall near the orifice plate. The test case has an internal diameter of the orifice plate of $d=65 \mathrm{~mm}$ and a mean flow speed of $U=10.8 \mathrm{~m} / \mathrm{s}$ in the duct. 
Fig. 6. PSD of the pressure fluctuations on the upstream and downstream surface of the orifice plate along radial direction with an internal diameter of the orifice plate of $d=65 \mathrm{~mm}$ and a mean flow speed of $U=10.8 \mathrm{~m} / \mathrm{s}$ in the duct. (a) PSD of the pressure fluctuation on the upstream surface of the orifice plate. (b) PSD of the pressure fluctuation on the downstream surface of the orifice plate.

Fig. 7. Comparison of the PSD of the pressure fluctuation on the upstream surface of the orifice plate at a radius of $41.25 \mathrm{~mm}$ along different angular position $\theta$ with an internal diameter of the orifice plate of $d=65 \mathrm{~mm}$ and a mean flow speed of $U=10.8$ $\mathrm{m} / \mathrm{s}$ in the duct.

Fig. 8. Coherence of the surface pressure with different separation distance on the upstream side of the orifice plate with an internal diameter of the orifice plate of $d=65$ $\mathrm{mm}$ and a mean flow speed of $U=10.8 \mathrm{~m} / \mathrm{s}$ in the duct. For radial direction, the coherence was measured between pressure tap $T_{9}$ and other pressure taps in radial direction. For circumferential direction, the coherence was between pressure tap $T_{19}$ and other pressure taps along circumferential direction. (a) Coherence of the surface pressure on the upstream side along radial direction. (b) Coherence of the surface pressure on the upstream side along circumferential direction.

Fig. 9. Coherence of the surface pressure with different separation distance on the downstream side of the orifice plate with an internal diameter of the orifice plate of $d=65 \mathrm{~mm}$ and a mean flow speed of $U=10.8 \mathrm{~m} / \mathrm{s}$ in the duct. For radial direction, the coherence was between pressure tap $T_{1}$ and other pressure taps in radial direction. For circumferential direction, the coherence was between pressure tap $T_{3}$ and other pressure taps along circumferential direction. (a) Coherence of the surface pressure on the downstream side along radial direction. (b) Coherence of the surface pressure on the downstream side along circumferential direction.

Fig. 10. CPSD of the pressure fluctuation on the upstream surface of the orifice plate along radial direction and circumferential direction with an internal diameter of the orifice plate of $d=65 \mathrm{~mm}$ and a mean flow speed of $U=10.8 \mathrm{~m} / \mathrm{s}$ in the duct. (a) Cross spectral density (CPSD) of the pressure fluctuation on the upstream surface of the orifice plate along radial direction. (b) Cross spectral density (CPSD) of the 
pressure fluctuation on the upstream surface of the orifice plate along circumferential direction.

Fig. 11. Illustration of the approximate calculation of the surface pressure cross spectrum density between any two points.

Fig. 12. The comparison of PWL spectra from far field measurements and surface pressure measurements for the orifice plate with an internal diameter of $d=65 \mathrm{~mm}$ under different mean flow speeds in the duct.

Fig. 13. The average coherence area in plane wave frequency range for the orifice plate with an internal diameter of $d=65 \mathrm{~mm}$ under different mean flow speeds in the duct.

Fig. 14. Different velocity scaling laws for sound power, surface pressure and correlation area, $\Omega$ represents sound power, sound pressure and correlation area integrated over 500-2000 Hz respectively.

Fig. 15. Sound source distribution on the surface of the orifice plate for the plane wave. The test case is for an internal diameter of $65 \mathrm{~mm}$ under a mean flow speed of $9.9 \mathrm{~m} / \mathrm{s}$. 